\title{
Introduction:
}

Kleine-Levin Syndrome (KLS) is described as a rare disorder presenting with recurrent episodes of unexplained hypersomnia lasting from few days to several weeks, hyperphagia and hypersexuality. It affects mainly young male individuals. The cause of KLS is not known, but evidence suggests it could be a recurrent inflammatory encephalitis. Between episodes, subjects don't show any symptom and sometimes describe retrograde amnesia for their behavior. Nevertheless, and since this disorder can cause a great impairment on social and working abilities during episodes, we can observe a significant prevalence of depression and anxiety among patients as a consequence to their clinical situation.

\section{Clinical Case:}

Identification and Medical History

- Male

- 51 years old

- Married

- Living with wife and his daughter, with 23 years old

- Unemployed, no occupation

- Medical history without relevance

- No history of epilepsy

- Psychiatric and neurological

follow up since 2017

- Valproic acid 500mg twice/day

- No previous history of hospital admissions

\section{Clinical Features}

- Was observed on Neurology Emergency Room in December 2018 from Centro Hospitalar de Trás-os-Montes e Alto Douro

- Presented with disorientation, sexual impulsivity, aggressiveness, hypersomnia and hyperphagia from the last few days

- His wife and daughter had already left home due to his behavioral changes

- Neurological examination normal

- No previous history of trauma

- Laboratorial findings: valproic acid below therapeutic cut-off
Treatment and Evolution

- Admitted at Psychiatric ward in order to optimize the pharmacotherapy and stabilize the patient

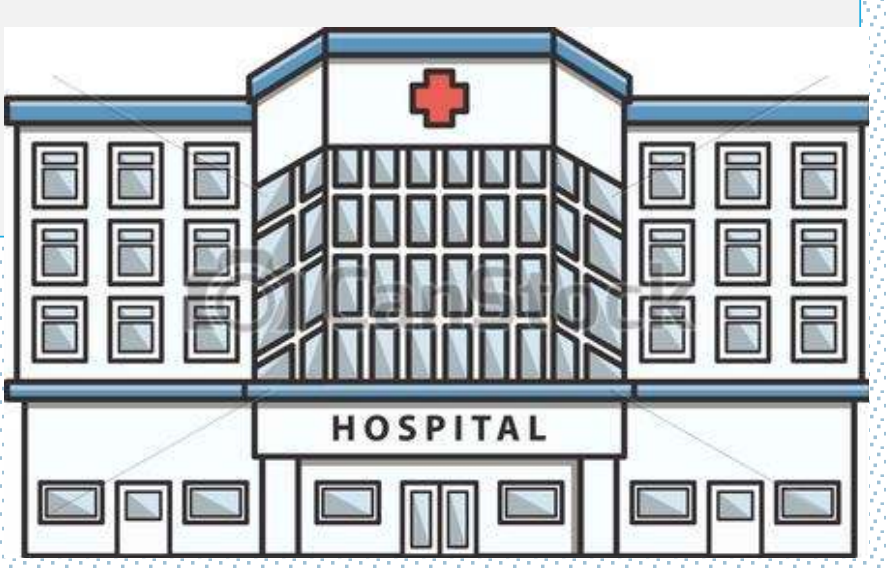

\section{Conclusions:}

Despite being rare, KLS can lead to great changes on personal, social and working life of our patients. Besides, this condition can be related to the emergence of depressive and anxious symptoms that must be adequately treated. For all these reasons, it's important to approach this theme in order to raise the awareness of its existence and improve its diagnosis and treatment.

\section{Biography}

Kleine-Levin syndrome: Etiology, diagnosis, and treatment, Santosh Ramdurg, Ann Indian Acad Neurol. 2010 Oct-Dec; $13(4): 24$ 1-246.

The Kleine-Levin Syndrome: A Rare Disease with Often Delayed Diagnosis-A Report of Two Cases in the Department of Neurology of the University

Hospital of Cocody (Côte d'Ivoire), Berthe Assi, * Constance Yapo-Ehounoud, Mohamed Ben Allaoui Baby,Evelyne Aka-Diarra, Muriel Amon-

Tanoh, and Christian Tanoh, Case Rep Neurol Med. 2016; 2016: 8929413.

Kleine-Levin syndrome: A neuropsychiatric disorder. 